\title{
Olympic Medals: Does The Past Predict The Future?
}

\author{
Yi Han \\ School of Electrical \& Electronic Engineering, North China Electric Power University, Baoding \\ 071003, China \\ m18636256181@163.com
}

Keywords: Gray Prediction Model, Olympic medal, Historical Achievements.

\begin{abstract}
With the United States retain its position as the top medal-winning nation at last year's Olympic Games in Rio, the most greatly desirable event came to the end. Is the result the same as your expectation? Is it possible to predict how many medals each nation will win? And what is it about a nation that allows it to produce Olympic medal-winning athletes? Even though the performances of individual athletes can vary unpredictably, we reasoned, there might be an overall relationship between a country's fundamental characteristics (its size and amount of wealth, for instance) and the number of medals it would likely take home. First, considering the past Olympic success, we developed a factor model with its weight in achievement of Games calculated by Grey Prediction Model. Based on this model, we chose china for further analysis and found that there is a link between them but not obvious.
\end{abstract}

\section{Introduction}

Olympics, hold once every four years, are currently the world's most influential sports event. In the Olympic Games, countries exchange cultures and swap experience and skills. The aim is to encourage people to keep on sport. [1] During the Olympic Games, medal table attracts world attention. The Rio Olympics have come to the end, and of one thing we can be confident - the US and China showed their powerful actual strength once more. But why are they always so successful? And can we predict which other countries will do well the next Olympics? [2]

If we look at the medal counts for the two most recent Olympic Games (see Table 1), we see that the top two nations are the U.S. and China, who happen to be the 3rd and 1st most populous nations in the world. So population seems to be important. But where is India, the world's second most populous nation? Maybe wealth is the key factor. That seems to fit. A lot of the nations at the top of the list are the wealthier nations of the world. But how did Cuba and Belarus rank so high? As we think more and more about it, it quickly becomes clear that the why's behind the medal counts at the Olympics are complex.

Table 1 Olympic medals

\begin{tabular}{|clcr|}
\hline \multirow{2}{*}{ Rank } & Nation & $\frac{\text { OTympic Meda7s }}{2004}$ & $\frac{07 y m o i c}{2008}$ \\
1 & United States & 103 & 110 \\
2 & China & 63 & 100 \\
3 & Russia & 92 & 72 \\
4 & United Kingdom & 30 & 47 \\
5 & Australia & 49 & 46 \\
6 & Germany & 49 & 41 \\
7 & France & 33 & 40 \\
8 & Korea, South & 30 & 31 \\
9 & Italy & 32 & 28 \\
10 & Ukraine & 23 & 27 \\
11 & Japan & 37 & 25 \\
12 & Cuba & 27 & 24 \\
13 & Belarus & 15 & 19 \\
14 & Canada & 12 & 18 \\
15 & Spain & 19 & 18 \\
$\cdots$ & ... & & \\
\hline
\end{tabular}


In order to make the prediction more accurate, we first discuss laterally the historical results influence of Olympic Games. Then take Chinese as an example to analyze the influence

\subsection{Symbol Description}

\begin{tabular}{ll}
\hline \hline Symbol & Description \\
$x^{(0)}$ & The original sequence of the amount predicted by the model. \\
$\hat{x}^{(0)}$ & The prediction of the amount of simulated sequence \\
$\mathrm{x}(0,0)$ & Observation series \\
$a$ & Development of gray scale \\
$u$ & Endogenous gray level \\
$\Delta$ & Average relative error \\
$\varepsilon(0)$ & Residual series \\
$\varepsilon(k)$ & relative error \\
$C$ & Posterior difference ratio \\
$Y$ & Olympic medals \\
$\mathrm{y}$ & Winning rate \\
$P$ & probability of small error \\
$R^{2}$ & determinant coefficient \\
\hline \hline
\end{tabular}

\section{Model}

In this section, we construct a model capable of analyzing the factors that influence Olympic medal.

\subsection{Historical Achievements Model}

First, we approached the problem by building a factor model with its weight calculated by grey Relational Analysis.

\subsubsection{Model Introduction}

Gray Prediction Theory, based on the Gray System Theory, established by Chinese Scholar Professor Deng Julong in 1982, is a new method to solve the problems that are lack of data and information. It is widely used in random and orderly gray forecasting process, thus to find its potential patterns. [3] In this paper, based on the available data and uncertain information, the gray prediction method established a $\operatorname{GM}(1,1)$ model from the past extended to the future, in order to determine the trend of system development and provide the basis for projecting and policy-making. The randomness is weakened and the uncertainty is enhanced in the grey prediction model, as predicting the number on the size of time series. Meanwhile, at a deeper level, solve the function, Based on which, the prediction sequence is established and the prediction model is first-order differential equations.

Set $\mathrm{X}(0,0)$ as the raw data sequence, and

$$
x^{(0)}=\left\{x^{(0)}(1), x^{(0)}(2), \ldots x^{(0)}(n)\right\}
$$

Using 1-AGO to generate a first order cumulative generation sequence

$$
\begin{aligned}
& x^{(1)}=\left\{x^{(1)}(1), x^{(1)}(2), \ldots x^{(1)}(n)\right\}, \\
& x^{(1)}(k)=\sum_{i=1}^{k} x^{(0)}(i)
\end{aligned}
$$

The differential equation is established for the generation sequence $x^{(1)}(k)$

$$
\frac{d x^{(1)}}{d t}+a x^{(1)}=u
$$

a stand for development of gray scale

$\mathrm{u}$ stand for endogenous gray level

We can estimate the parameter a, $u$ by the least square method, construct the data matrix $\mathrm{B}$ and vector $\mathrm{Y}$ as follow: 


$$
\hat{x}^{(1)}(k+1)=\left(x^{(0)}(1)-\frac{u}{a}\right) e^{-a k}+\frac{u}{a}
$$

The $\hat{x}^{(1)}$ were down reduction from the original series fitting sequence

$$
\hat{x}^{(0)}(k+1)=\left(x^{(0)}(1)-\frac{u}{a}\right)\left(1-e^{a}\right) e^{-a k}
$$

\subsubsection{Assumption}

Based on this model, we chose china for further analysis. First we discuss the possible relevance between history records and this time. Hypothesizing that for a long time, the number of Chinese participants in Olympic Games will not make a larger change.

\subsubsection{Analysis and results}

Take the recent eight (25th, 26th, 27th, 28th, and 29th) Summer Olympic Games medals results as the object. The table below is the data we can compile.

Table 2 Olympic medals and medals in China

\begin{tabular}{c|cccccccc}
\hline \hline China & 1988 & 1992 & 1996 & 2000 & 2004 & 2008 & 2012 & 2016 \\
medals & 28 & 54 & 50 & 59 & 63 & 100 & 87 & 70 \\
ranking & 11 & 4 & 4 & 3 & 2 & 1 & 2 & 3 \\
percentage & 0.0387 & 0.0698 & 0.0615 & 0.0653 & 0.0695 & 0.1104 & 0.0960 & 0.0772 \\
\hline \hline
\end{tabular}

Winning percentage $=$ the number of medals/total medals

We can get the raw data sequence from Table 1 as follow:

$x^{(0)}=\left\{\begin{array}{llllllll}0.0387 & 0.0698 & 0.0615 & 0.0653 & 0.0695 & 0.1104 & 0.0960 & 0.0772\end{array}\right\}$

Using grey system prediction model, the obtained fitting sequence equation is

$x^{[1]}(\mathrm{k}+1)=\left(x^{(0)}(1)-\frac{0.0610}{-0.0595}\right) e^{0.0595 k}+\frac{0.0610}{-0.0595}$

$x^{[0]}(\mathrm{k}+1)=x^{(\mathbb{1})}(\mathrm{k}+1) x^{(\mathbb{1})}(\mathrm{k})$

$x^{(1)}(\mathrm{k}+1)=\left(x^{(0)}(1)-\frac{0.0610}{-0.0595}\right) e^{0.0595 k}-\left(x^{(0)}(1)-\frac{0.0610}{-0.0595}\right) e^{0.0595(k-1)}$

Based on Grey Prediction, forecasting sequence is:

$x^{(1)}=\left\{\begin{array}{llllllll}0.0653 & 0.0693 & 0.0735 & 0.0780 & 0.0828 & 0.0879 & 0.0933 & 0.0990\end{array}\right\}$

The establishment of the $\operatorname{GM}(1,1)$ of the gold-medal results of Olympic Games:

Table 3 the result of forecast

\begin{tabular}{c|cccccccc}
\hline \hline china & 1988 & 1992 & 1996 & 2000 & 2004 & 2008 & 2012 & 2016 \\
percentage & 0.0387 & 0.0698 & 0.0615 & 0.0653 & 0.0695 & 0.1104 & 0.0960 & 0.0772 \\
Forecasting percentage & 0.0635 & 0.0693 & 0.0735 & 0.0780 & 0.0828 & 0.0879 & 0.0933 & 0.0990 \\
relative error $\boldsymbol{\varepsilon}(\mathbf{k})$ & -0.0248 & 0.0005 & -0.012 & -0.0127 & -0.0133 & 0.0225 & 0.0027 & -0.0281 \\
\hline \hline
\end{tabular}

Besides, posterior difference ratio $\mathrm{C}=s_{2}=0.6260$, Small probability error $\mathrm{P}=0.75$, Correlation degree is $63.29 \%$.

According to the error level:

Table 4 the level of model

\begin{tabular}{c|c|c|c}
\hline \hline grade & relative error $\mathrm{q}$ & variance ratio C & Small probability error $\mathrm{P}$ \\
\hline I & $<0.01$ & $<0.35$ & $>0.95$ \\
\hline II & $<0.05$ & $<0.50$ & $<0.80$ \\
\hline III & $<0.10$ & $<0.65$ & $<0.70$ \\
\hline IV & $>0.20$ & $>0.80$ & $<0.60$ \\
\hline
\end{tabular}

So the model level is 3(Barely qualified), which illustrates, for percentage of medals, the relationship between the history of Olympic Games and the Olympic Games in China this time without considering any other factors is 


$$
x^{(1)}(\mathrm{k}+1)=\left(x^{(0)}(1)-\frac{0.0610}{-0.0595}\right) e^{0.0595 k}-\left(x^{(0)}(1)-\frac{0.0610}{-0.0595}\right) e^{0.0595(k-1)}
$$

To predict more accurately, other factors need to be taken into account.

\section{Conclusion}

Numerous factors, ranging from the physical to the political and socioeconomic, affect whether a country performs well during the Olympic Games. Among these are national power, population, economic status, professional capacity (including education and working conditions), political issues, social issues, and so on.

\section{References}

[1] Baidubaike, Retrieved from: https://en.wikipedia.org/wiki/ Economic Olympics

[2] Predicting the Rio Olympic medal table: http://www.bbc.com/news/magazine-36955132

[3] http://www.topendsports.com/events/summer/medal-tally/rankings-predicted.htm

[3] Xu Jianhua. Mathematical Methods in Modern Geography [M], Beijing: Higher Education Press, 2002.

[4] Liu Di. Brief Discussion on Gray Model and Its Application in Physical Education [J], Fujian Sports Science, 2002, 21(1): 6-8

[5] HuaLong Yang, Jinxia Liu, Bin Zheng, Improvement ang Application Of Grey Prediction GM(1,1) Model,Mathematics in Practice and Theory, 2011.(23): 1000-0984

[6] http://www.huffingtonpost.com/news/olympics-medal-predictions/

[7] Latitude: http://www.discoverycorpsinc.com/predicting-the-olympic-medal-c/ 